\title{
Calcium Score of Iliac Vessels and Relative Sparing of the External Iliac Artery Could Recommend Surgical Arterial Graft Selection
}

\author{
Pooya Torkian ${ }^{1}$, Taraneh Faghihi Langroudi ${ }^{1,}{ }^{*}$, Kobra Eslami ${ }^{1}$, Abbas Arjmand Shabestari ${ }^{1}$ and Reza \\ Talaie $^{2}$ \\ ${ }^{1}$ Department of Radiology, Shahid Beheshti University of Medical Sciences, Tehran, Iran \\ ${ }^{2}$ Department of Radiology, University of Minnesota, Minneapolis, United States \\ "Corresponding author: Department of Radiology, Shahid Beheshti University of Medical Sciences, Tehran, Iran. Tel: +98-9123509221, Email: faghihit@yahoo.com \\ Received 2019 March 08; Revised 2019 December 03; Accepted 2019 December 15.
}

\begin{abstract}
Background: Calcified plaque build-up can increase the risk of subsequent vascular complications in pelvic solid organ transplantation and vascular bypass procedures.

Objectives: To assess the atherosclerotic calcification of pelvic vessels by measuring Agatston score and comparing it among abdominal aorta, common iliac artery (CIA), external iliac artery (EIA), and common femoral artery (CFA).

Patients and Methods: We retrospectively reviewed the unenhanced computed tomography scans of 197 patients from August 2016 to March 2018. Using calcium-scoring software, we examined the distribution map of calcified plaques, focusing on four different arterial segments -the abdominal aorta, the common iliac artery (CIA), the external iliac artery (EIA), and the common femoral artery (CFA)- to determine which one showed the least mural calcification.

Results: A total of 197 patients (118 men, 79 women) with a mean age of $61.19 \pm 10.8$ years were included in this study. The right EIA segment had the lowest average calcification score ( $92.1 \pm 18.2$ ), including 180 cases (91.4\%) of zero calcification, followed by 178 cases (90.4\%) of zero calcification in the left EIA. When adjustments were made for age, gender, smoking status, diabetes, hypertension and hyperlipidemia, the odds ratio (OR) of the left EIA for reaching a higher calcium score level was $1.19(\mathrm{P}=0.44)$ times greater than the reference group (right EIA). The ratio was markedly higher in the abdominal aorta segment $(\mathrm{OR}=146.7 .4, \mathrm{P}<0.001)$, followed by the right $\mathrm{CIA}(\mathrm{OR}=42.5, \mathrm{P}<0.001)$, the left $\mathrm{CIA}(\mathrm{OR}=27.3, \mathrm{P}<0.001)$, the right $\mathrm{CFA}(\mathrm{OR}=3.96, \mathrm{P}<0.001)$, and the left $\mathrm{CFA}(\mathrm{OR}=$ $3.51, \mathrm{P}<0.001)$.
\end{abstract}

Conclusion: The study demonstrates a significantly less calcific plaque burden in EIA compared to the adjacent arterial bed.

Keywords: External Iliac Artery, Calcified Plaque, Atherosclerosis, Arterial Graft

\section{Background}

Atherosclerosis, or the hardening of arteries, usually manifests with mural calcification (1). Vascular calcific deposition has long been implicated in the formation of atherosclerosis. The vascular calcification in the intimal or medial layers of the arterial wall is routinely detected. Many studies have indicated that calcified plaque buildup, termed arterial calcification, could be associated with technical failure of arterial anastomosis, delayed graft function (DGF), subsequent vascular morbidity analogous to steal syndrome and risk of operative complexity $(2,3)$. Arterial calcification is quantified by a measurement technique called the Agatston score and has a substantial effect on patient survival $(4,5)$. Over the past few years, we noticed a relative atherosclerotic sparing of the external iliac artery (EIA) compared to adjacent arteries. This is not a well-described phenomenon in the literature. Similar findings in other vessels have been attributed to the histological features of the wall or its anatomical feature (6).

\section{Objectives}

This study was designed to test our hypothesis that fewer atherosclerosis changes occur in the EIA than that of adjacent arteries. If true, this could have significant implications on vascular and transplant surgery, for example in the choice of arterial anastomosis in solid organ transplantation or bypass graft placement.

\section{Patients and Methods}

Institutional review board approval was obtained for collecting data and interpreting the analysis. The study 
protocol was approved by our institution's research ethics committee. In this study, 197 patients with non-contrastenhanced abdominopelvic computed tomography (CT) scan from August 2016 to March 2018 were retrospectively evaluated (Figure 1). A non-contrast-enhanced CT scan of the abdomen and pelvis was performed in patients with non-traumatic abdominal pain, suspected renal stones, and urologic disorders or fever of unknown origin (highly suspected to be of abdominal abscess). Image evaluations were performed simultaneously by an experienced cardiovascular radiologist. Patients with prior arterial stent implantation in the abdominal aorta, common iliac artery (CIA), external iliac artery (EIA), common femoral artery (CFA), a history of operation on the arteries of interest, a former pelvic surgery, presence of a severe motion artifact or metallic artifacts from adjacent prosthesis that preclude accurate interpretation (at radiologist's discretion) were deemed ineligible for our study.

Images from the abdominal and pelvic CT scans (Brilliance 64, Philips Medical Systems, Cleveland, Ohio) were retrospectively evaluated. Images were reconstructed with the following settings: collimation: $32 \times 1.25 \mathrm{~mm}$; gantry rotation time: $0.5 \mathrm{~ms}$; tube voltage: $160 \mathrm{kV}$; tube current: 390 mAs; and pitch: 0.97. Each complete scan contained 50 - 60 contiguous, non-overlapping, $512 \times 512$ matrix slices over a 35-cm field of view, with peak tube voltage of $160 \mathrm{kV}$ using 100-ms exposure time with 3-mm slice thickness. For purposes of this study, we defined abdominal aorta from the diaphragmatic level to the aortic bifurcation; common iliac artery (CIA) from the aortic bifurcation to the CIA bifurcation; EIA from the CIA bifurcation to the inguinal ligament; and common femoral artery (CFA) from the inguinal ligament to the CFA bifurcation. To determine the calcium score of the abdominal aorta, CIA, EIA, and CFA, calciumscoring software was used to measure the mean Agatston score, which is based on the weighted density score given to the highest attenuation value (Hounsfield unit [HU]) multiplied by the area of the calcified plaque. The mean Agatston score was calculated with semi-automated software (HeartBeat-CS, Brilliance Workspace, Brilliance 64, Philips Medical Systems) by using detection of calcified plaque with a threshold of $130 \mathrm{HU}$ and a minimum area of $1 \mathrm{~mm}^{2}$. Due to close proximity of internal iliac artery (IIA) to the sacral bone and calcification in its small branches, we could not measure the calcium score by our software meticulously.

\subsection{Statistical Analysis}

Generalized estimating equations (GEEs) were used to evaluate the age, gender, smoking, hypertension, diabetes and hyperlipidemia -adjusted effect of arterial anatomic position on the calcification level. The GEE procedure extends the generalized linear model to allow for the analysis of repeated measurements or other correlated observations. The use of this statistical method allows for the use of other vessels in each patient as the internal control. This will control the effect of confounders. Calcification score was considered at four levels: 0, 1 - 100, 101 - 400, and higher than 400. According to the calcium measurement scale, cumulative logistic function in GEE was used to estimate the parameters. Sub-group analysis was performed in order to determine the validity of the findings in patients with more severe atherosclerotic disease. Odds ratios (ORs) and 95\% confidence intervals (CIs) of calcium scoring were examined for the aforementioned four arterial anatomic positions.

\section{Results}

A total of 197 patients (118 men, 79 women) with a mean age of $61.19 \pm 10.8$ years were included in the study. Analysis of the calcium score levels across the abdominal aorta, CIA, EIA, and CFA is presented in Table 1 and Figure 2. It revealed 180 cases (91.4\%) of zero calcification in the right EIA, followed by 178 cases (90.4\%) of zero calcification in the left EIA. High calcium score level (> 400) was seen in 108 (54.8\%) cases of abdominal aorta segments, which were disproportionately higher than other segments. The average calcification score was the lowest in the right EIA segment (18.21), which was selected as the reference group (Figure3). Based on GEE results (Table 2), the OR of the left EIA reaching a higher calcium score level was 1.19 (95\% CI = 0.77-1.84, $\mathrm{P}=0.44$ ) times greater than the reference group (right EIA). This ratio was markedly higher $(\mathrm{OR}=146.7,95 \% \mathrm{CI}=79.9$ 249.0, $\mathrm{P}<0.001)$ in the abdominal aorta segment, followed by $\mathrm{OR}=42.5$ (95\% $\mathrm{CI}=23.4-77.2, \mathrm{P}<0.001)$ in the right CIA, $\mathrm{OR}=27.3(95 \% \mathrm{CI}=15.7-47.6, \mathrm{P}<0.001)$ in the left CIA, OR= $3.96(95 \% \mathrm{CI}=2.38-6.61, \mathrm{P}<0.001)$ in the right CFA, and $3.51(95 \% \mathrm{CI}=2.11-5.85, \mathrm{P}<0.001)$ in the left CFA, when adjustments were made for age, gender, smoking status, hypertension (HTN), diabetes mellitus (DM) and hyperlipidemia status in the GEE model. Subgroup analysis of patients with severe atherosclerotic disease (median abdominal aortic calcium score more than 493) revealed no significant difference in relative sparing of EIA when compared to the subgroup with the least severe atherosclerotic disease (median abdominal aortic calcium score less than 493) (P $=0.99$ ).

\section{Discussion}

There is a paucity of studies showing sparing calcification in EIA despite gross calcifications in different adjacent 
266 patients were referred to the imaging department for doing non-contrast enhanced abdomino-pelvic CT scan

Exclusions:

-Prior stent implantation

$(\mathrm{N}=14)$

-History of operation on

those arteries $(\mathrm{N}=13)$

-Former pelvic surgery

$(\mathrm{N}=20)$

-Presence of a severe

motion $\operatorname{artifact}(\mathrm{N}=22)$

Images of 197 patients with non-traumatic

abdominal pain, suspected renal stones,

urologic disorders or fever of unknown

origin were evaluated.

Figure 1. Flow chart of the study population selection including patient recruitment and exclusion criteria

Table 1. Frequency (Percent) of Calcification Level Across Different Arterial Segments

\begin{tabular}{|c|c|c|c|c|c|c|}
\hline \multirow{2}{*}{ Artery } & \multicolumn{4}{|c|}{ Calcification level, frequency (percent) } & \multicolumn{2}{|c|}{ Calcium score } \\
\hline & $\mathbf{0}$ & $1-100$ & $101-400$ & $>400$ & Median $(\min -\max )$ & Mean $\pm S D$ \\
\hline Aorta & $4(2.0)$ & $39(19.8)$ & $46(23.4)$ & $108(54.8)$ & $493.3(0-13140)$ & $1235.8 \pm 1828$ \\
\hline CIA right & $57(28.9)$ & $37(18.8)$ & $36(18.3)$ & $67(34.0)$ & $113.4(0-7263)$ & $436.1 \pm 798$ \\
\hline CIA left & $68(34.5)$ & $43(21.8)$ & $38(19.3)$ & $48(24.4)$ & $44.4(0-5906)$ & $334.7 \pm 697$ \\
\hline EIA right & $180(91.4)$ & $8(4.1)$ & $5(2.5)$ & $4(2.0)$ & $0(0-763)$ & $18.2 \pm 92.1$ \\
\hline EIA left & $178(90.4)$ & $6(3.0)$ & $8(4.1)$ & $5(2.5)$ & $0(0-1432)$ & $26.1 \pm 136.2$ \\
\hline CFA right & $146(74.1)$ & $27(13.7)$ & $19(9.6)$ & $5(2.5)$ & $0(0-1544)$ & $43.4 \pm 147$ \\
\hline CFA left & $149(75.6)$ & $27(13.7)$ & $17(8.6)$ & $4(2.0)$ & $0(0-1383)$ & $44.3 \pm 141$ \\
\hline
\end{tabular}

Abbreviations: CFA, common femoral artery; CIA, common iliac artery; EIA, external iliac artery; SD, standard deviation

vascular segments, abdominal aorta, CIA, and CFA. We examined the distribution map of calcified plaque, focusing on four different arterial segments to determine the lowest calcification score among them and we found out that EIA shows a significantly lower degree of atherosclerotic calcification compared to abdominal aorta, CIA and CFA. The importance of this finding may influence decisions for the usefulness of harvested EIA as an allograft in place of synthetic grafts or xenografts, which are currently used for some cases who are in need of a vascular graft.

Conduit calcification and stenosis is one of the compli- cations in the placement of right ventricle to pulmonary artery conduit (7). Previously, the pulmonary homograft (PH) was selected mostly for the right ventricle to pulmonary artery (RV-PA) conduit (8). However, calcification and stenosis with deterioration of valve function have been shown in different studies (8-10). Amongst various conduit materials, the expanded polytetrafluoroethylene (ePTFE) has yielded minimal rates of complications including conduit stenosis, conduit regurgitation and conduit reoperation $(8,11)$. Despite its benefits, the availability of ePTFE could be limited at small institutions. Based on our 


\begin{tabular}{|c|c|c|c|c|}
\hline \multirow{2}{*}{ Artery $^{c}$} & \multicolumn{2}{|c|}{ Compared to EIA right } & \multicolumn{2}{|c|}{ Compared to EIA left } \\
\hline & $\mathrm{OR}(95 \% \mathrm{CI})$ & Pvalue & OR $(95 \% \mathrm{CI})$ & Pvalue \\
\hline Aorta & $146.7(79.9-249.0)$ & $<0.001$ & $123.4(69.5-219.2)$ & $<0.001$ \\
\hline CIA right & $42.5(23.4-77.2)$ & $<0.001$ & $35.7(20.8-61.4)$ & $<0.001$ \\
\hline CIA left & $27.3(15.7-47.6)$ & $<0.001$ & $23.0(13.6-38.8)$ & $<0.001$ \\
\hline EIA right & 1 & & $0.84(0.54-1.30)$ & 0.44 \\
\hline EIA left & $1.19(0.77-1.84)$ & 0.44 & 1 & \\
\hline CFA right & $3.96(2.38-6.61)$ & $<0.001$ & $3.33(2.09-5.31)$ & $<0.001$ \\
\hline CFA left & $3.51(2.11-5.85)$ & $<0.001$ & $2.96(1.88-4.66)$ & $<0.001$ \\
\hline
\end{tabular}

Abbreviations: CFA, common femoral artery; CIA, common iliac artery; EIA, external iliac artery

${ }^{a}$ The effects of age, gender, smoking status, hypertension, diabetes and hyperlipidemia were adjusted in GEE model

${ }^{b}$ Odds ratio(OR) and 95\% confidence interval (CI) of calcification in different arterial segments were determined based on an ascending succession in the arterial system.

'The Wald's chi-square value for artery variable was $418.20, \mathrm{DF}=6$ and $\mathrm{P}<0.001$.

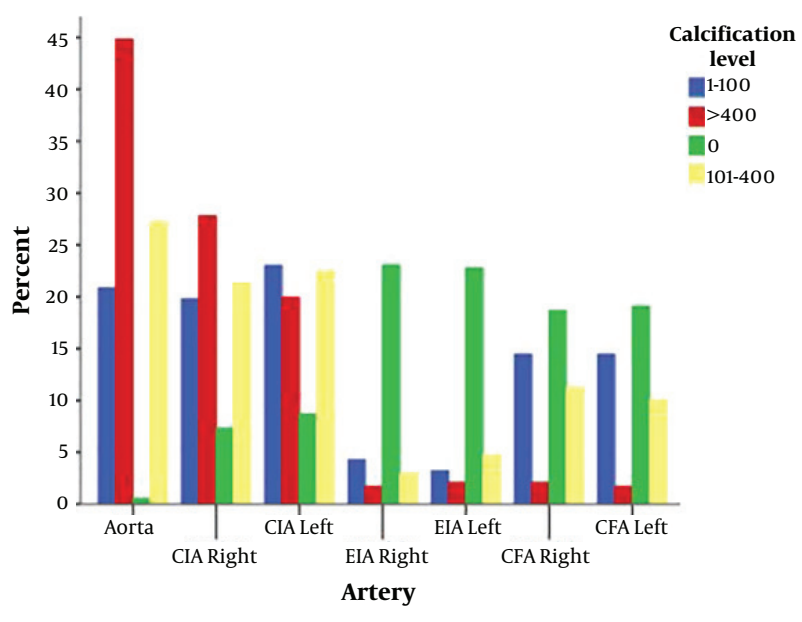

Figure 2. Different calcification levels among different arterial segments. CFA, common femoral artery; CIA, common iliac artery; EIA, external iliac artery

findings, EIA could be used as a graft target due to its less calcific plaque burden compared to the adjacent arterial bed. There is scant evidence of using EIA as a conduit placement option. It may be worthy to plan a study for harvesting EIA as cadaver arterial allograft for placement of the right ventricle to pulmonary artery conduit with regards to relative atherosclerotic sparing of the EIA.

Additionally, future studies could be performed in order to demonstrate EIA as a less vasculopathic arterial graft target in different regions. This can be done by end-to-end anastomosis to the patent part and replacing the occluded section by EIA.

It is well established that vascular calcification is the main cause of multiple complications in renal transplant surgeries, including the process of anastomosis, difficulty

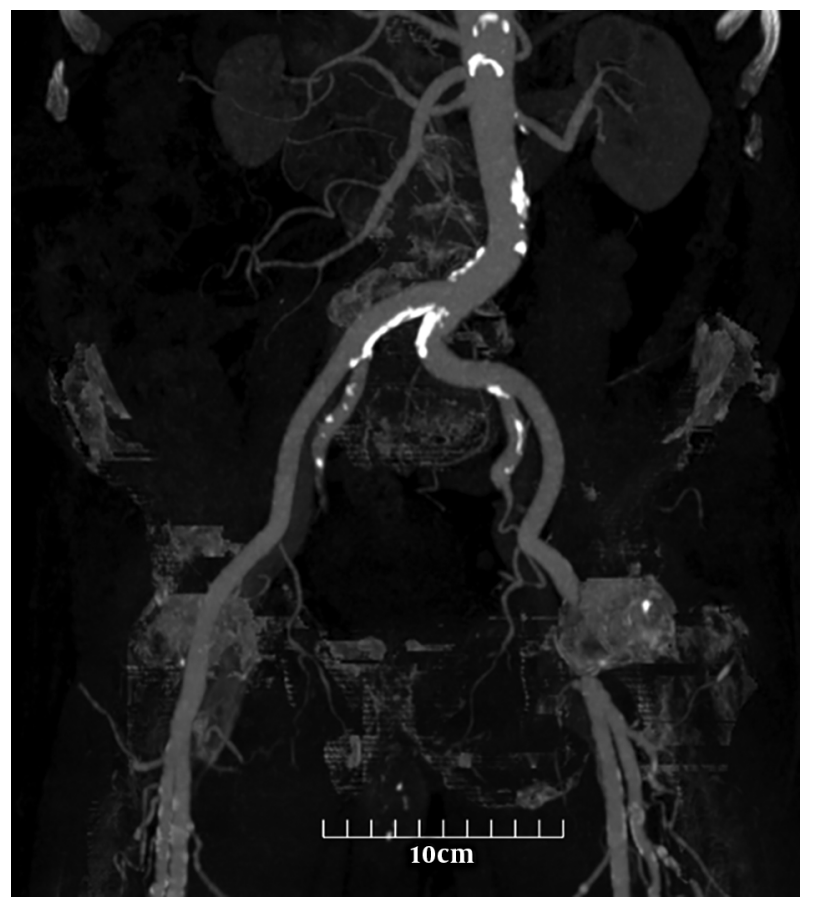

Figure 3. A 56-year-old man with no significant medical history presented with complaints of non-traumatic abdominal pain. To evaluate a patient with suspected vascular disease, a noncontrast computed tomography scan of the abdomen and pelvis was performed. Less calcification formation in the external iliac artery compared to adjacent arteries is seen. The calcium content of each artery was calculated with semi-automated software. The scores are as follows: Aorta: 6779.8, right common iliac artery (CIA): 1016.5, left CIA: 290.1, right external iliac artery (EIA):0, left EIA:0, right common femoral artery (CFA):548.7, and left CFA: 416.9 .

in vessel clamping, impaired graft function, and the occurrence of arterial steal syndrome or limb ischemia (12). Most practices require an entity of vascular imaging (usually an ultrasound) before deciding the proper site for anastomo- 
sis of the new implant, in part to assess the size and health of patent arteries. Calcification can occur in either the intimal or medial layer of the arteries (13). Intimal calcifications can be accelerated by advanced age, diabetes, and the presence of atherosclerosis; however, medial calcification can be found in people of younger age and in those who are on dialysis therapy (3). Aitken et al. showed high intraoperative complication rates and delayed graft function in patients with moderate to severe vascular calcifications (12).

The presence of vascular calcification has an impact on fusion and anastomosis events, which exacerbates the condition. Agatston score as an additional prognostic factor can describe arterial calcification. Therefore, careful evaluation and scoring of vascular calcification based on Agatston score preceding surgery may facilitate surgical intervention and improve efficacy and outcomes.

At our institution, most of the renal transplantations are performed by end-to-end anastomosis to the internal iliac artery (IIA). Previously, some studies favored EIA endto-side anastomosis over other arteries in renal transplantation (14). This finding could be attributed to larger caliber, existence of accessory renal arteries, and lower risk of compromising distal vascular supply to the pelvis $(14,15)$. However, Matheus et al. called this finding into question by showing no differences in clinical or surgical complications for arterial anastomosis between end-to-side anastomosis to the EIA and end-to-end anastomosis to the IIA(16).

This study compares calcification plaque in different segments of pelvic arteries. Our findings suggest that EIA can possibly be a less vasculopathic target for arterial grafts.

Our study was limited by its retrospective design and its small sample size, which reduced its precision. Therefore, future studies with larger sample sizes, more detailed evaluation of patient's history and CT angiography as a better choice to define plaque burden will be necessary for better understanding of this phenomenon and its etiology.

In conclusion, EIA has a significantly lower degree of atherosclerotic calcification compared to the adjacent vasculature. This can prove a useful alternative in selecting a less diseased target for arterial graft and pelvic solid organ arterial anastomoses.

\section{Footnotes}

Authors' Contributions: Taraneh Faghihi Langroudi chose the concept and design of the subject, Pooya Torkian and Kobra Eslami gathered clinical data and were responsible for general writing, clinical imaging and diagnosis, and Abbas Arjmand Shabestari and Reza Talaie revised the manuscript.
Conflict of Interests: The authors have no conflicts of interest to declare.

Ethical Approval: This was a retrospective study. The institutional review board in our unit approved this retrospective study. The thesis was approved with publication number (\#IR.SBMU.MSP.REC.1397.142) at Shahid Beheshti University.

Funding/Support: There was no funding/support for the study.

Informed Consent: Written informed consent was obtained from all patients in this study.

\section{References}

1. Yahagi K, Kolodgie FD, Lutter C, Mori H, Romero ME, Finn AV, et al. Pathology of human coronary and carotid artery atherosclerosis and vascular calcification in diabetes mellitus. Arterioscler Thromb Vasc Biol. 2017;37(2):191-204. doi: 10.1161/ATVBAHA.116.306256. [PubMed: 27908890]. [PubMed Central: PMC5269516].

2. Davis B, Marin D, Hurwitz LM, Ronald J, Ellis MJ, Ravindra KV, et al. Application of a novel CT-based iliac artery calcification scoring system for predicting renal transplant outcomes. AJR Am J Roentgenol. 2016;206(2):436-41. doi: 10.2214/AJR.15.14794. [PubMed: 26797375].

3. Thompson T, Shields KJ, Barinas-Mitchell E, Newman A, SuttonTyrrell K. Calcified carotid artery plaques predict cardiovascular outcomes in the elderly. J Hypertens. 2015;33(4):810-7. discussion 817. doi: 10.1097/HJH.0000000000000488. [PubMed: 25915886].

4. Criqui MH, Denenberg JO, McClelland RL, Allison MA, Ix JH, Guerci A, et al. Abdominal aortic calcium, coronary artery calcium, and cardiovascular morbidity and mortality in the Multi-Ethnic Study of Atherosclerosis. Arterioscler Thromb Vasc Biol. 2014;34(7):1574-9. doi: 10.1161/ATVBAHA.114.303268. [PubMed: 24812323]. [PubMed Central: PMC4153597].

5. Williams MC, Murchison JT, Edwards LD, Agusti A, Bakke P, Calverley PM, et al. Coronary artery calcification is increased in patients with COPD and associated with increased morbidity and mortality. Thorax. 2014;69(8):718-23. doi: 10.1136/thoraxjnl-2012-203151. [PubMed: 24473329].

6. Davierwala PM, Mohr FW. Bilateral internal mammary artery grafting: Rationale and evidence. Int J Surg. 2015;16(Pt B):133-9. doi: 10.1016/j.ijsu.2015.01.012. [PubMed: 25612853].

7. Karamlou T, Blackstone EH, Hawkins JA, Jacobs ML, Kanter KR, Brown JW, et al. Can pulmonary conduit dysfunction and failure be reduced in infants and children less than age 2 years at initial implantation? J Thorac Cardiovasc Surg. 2006;132(4):829-38. doi: 10.1016/j.jtcvs.2006.06.034. [PubMed: 17000294].

8. Shinkawa T, Chipman C, Bozzay T, Tang X, Gossett JM, Imamura M. Outcome of right ventricle to pulmonary artery conduit for biventricular repair. Ann Thorac Surg. 2015;99(4):1357-66. doi: 10.1016/j.athoracsur.2014.07.095. [PubMed: 25725927].

9. Salem AM. Right ventricle to pulmonary artery connection: Evolution and current alternatives. J Egyptian Soc Cardio-Thorac Surg. 2016;24(1):47-57. doi: 10.1016/j.jescts.2016.04.009.

10. Selamet Tierney ES, Gersony WM, Altmann K, Solowiejczyk DE, Bevilacqua LM, Khan C, et al. Pulmonary position cryopreserved homografts: Durability in pediatric Ross and non-Ross patients. J Thorac Cardiovasc Surg. 2005;130(2):282-6. doi: 10.1016/j.jtcvs.2005.04.003. [PubMed: 16077388].

11. Brown JW, Halpin MP, Rescorla FJ, VanNatta BW, Fiore AC, Shipley GD, et al. Externally stented polytetrafluoroethylene valved conduits for right heart reconstruction. An experimental comparison with 
Dacron valved conduits. J Thorac Cardiovasc Surg. 1985;90(6):833-41. [PubMed: 2933562].

12. Aitken E, Ramjug S, Buist L, Kingsmore D. The prognostic significance of iliac vessel calcification in renal transplantation. Transplant Proc. 2012;44(10):2925-31. doi: 10.1016/j.transproceed.2012.06.058. [PubMed: 23194999].

13. Kahn J, Ram LM, Eberhard K, Groselj-Strele A, Obermayer-Pietsch B, Muller H. Calcification score evaluation in patients listed for renal transplantation. Clin Transplant. 2017;31(3). doi: 10.1111/ctr.12888. [PubMed: 27988970].

14. Pal DK, Sanki PK, Roy S. Analysis of outcome of end-to-end and endto-side internal iliac artery anastomosis in renal transplantation:
Our initial experience with a case series. Urol Ann. 2017;9(2):166-9. doi: 10.4103/0974-7796.204176. [PubMed: 28479769]. [PubMed Central: PMC5405661].

15. Mohamed IH, Bagul A, Doughman T, Nicholson ML. Use of internal iliac artery as a side-to-end anastomosis in renal transplantation. Ann $R$ Coll Surg Engl. 2012;94(1):e36-7. doi: 10.1308/003588412X13171221499540. [PubMed: 22524924]. [PubMed Central: PMC3954224].

16. Matheus WE, Reis LO, Ferreira U, Mazzali M, Denardi F, Leitao VA, et al. Kidney transplant anastomosis: Internal or external iliac artery? Urol J. 2009;6(4):260-6. [PubMed: 20027554]. 